Internat. J. Math. \& Math. Sci.

Vol. 24, No. 7 (2000) 481-491

S0161171200002970

(c) Hindawi Publishing Corp.

\title{
GENERALIZATION OF THE FORMULA OF FAA DI BRUNO FOR A COMPOSITE FUNCTION WITH A VECTOR ARGUMENT
}

\author{
RUMEN L. MISHKOV
}

(Received 12 January 1999)

\begin{abstract}
The paper presents a new explicit formula for the $n$th total derivative of a composite function with a vector argument. The well-known formula of Faa di Bruno gives an expression for the $n$th derivative of a composite function with a scalar argument. The formula proposed represents a straightforward generalization of Faa di Bruno's formula and gives an explicit expression for the $n$th total derivative of a composite function when the argument is a vector with an arbitrary number of components. In this sense, the formula of Faa di Bruno is its special case. The mathematical tools used include differential operators, polynomials, and Diophantine equations. An example is shown for illustration.
\end{abstract}

Keywords and phrases. Differentiation theory, computational methods, classical combinatorial problems.

2000 Mathematics Subject Classification. Primary 58C20; Secondary 17-08, 05Axx.

1. Introduction. Sometimes, when analyzing $n$th order nonlinear systems or developing mathematical processes such as presenting the solution of a problem in the general $n$th order case, it is necessary to have an explicit formula for the $n$th derivative of a composite function with a vector argument whose components are arbitrary in number. A powerful tool for managing $n$th order tasks is the formula of Faa di Bruno giving an explicit expression for the $n$th total derivative of the scalar composite function $f(x(t))$ with a scalar argument $x(t)$. If $f, x$, and $t$ are scalars and $f(x(t))$ is a composite function for which all the necessary derivatives are defined, then the $n$th derivative of the function $f$ with respect to $t$, in accordance with the formula of Faa di Bruno, is

$$
f_{t}^{(n)}=\sum \frac{n ! f_{x}^{(k)}\left(x^{(1)}\right)^{k_{1}}\left(x^{(2)}\right)^{k_{2}} \cdots\left(x^{(n)}\right)^{k_{n}}}{k_{1} ! k_{2} ! \cdots k_{n} !(1 !)^{k_{1}}(2 !)^{k_{2}} \cdots(n !)^{k_{n}}}
$$

where $k=k_{1}+k_{2}+\cdots+k_{n}$ and the sum is over all nonnegative integer solutions of the Diophantine equation $k_{1}+2 k_{2}+\cdots+n k_{n}=n$. The following notation is used in (1.1): $f_{t}^{(n)}-n$th derivative of the function $f$ with respect to $t, f_{x}^{(k)}-k$ th derivative of the function $f$ with respect to $x, x^{(i)}$-ith derivative of the function $x$ with respect to $t$. The right-hand sides of (1.1) for $n=1,2,3, \ldots$ are known as Bell polynomials (Bell [1]). The formula (1.1) and the Bell polynomials show that any total derivative $f_{t}^{(n)}$ is a linear function of the partial derivatives

$$
f_{x}^{(k)}, \quad k=1,2, \ldots, n,
$$


which are multiplied by the structural coefficients

$$
\frac{n !}{k_{1} ! k_{2} ! \cdots k_{n} !(1 !)^{k_{1}}(2 !)^{k_{2}} \cdots(n !)^{k_{n}}},
$$

and the nonlinear functions of the derivatives $x^{(i)}, i=1,2, \ldots, n$,

$$
\left(x^{(1)}\right)^{k_{1}}\left(x^{(2)}\right)^{k_{2}} \cdots\left(x^{(n)}\right)^{k_{n}} \text {. }
$$

There are various proofs of formula (1.1) based on different approaches-Taylor series representation (Jordan [4]), Bell polynomials (Comtet [3]), umbral calculus (Riordan [5] and Roman [6]).

The application of formula (1.1) is in tasks where an explicit formula for the $n$th derivative of the composite function with a scalar argument $f(x(t))$ has to be used, so it is restricted to the cases when the argument $x(t)$ is a scalar. The fact that the formula of Faa di Bruno considers only one scalar argument $x(t)$ in the expression for the $n$th derivative of the composite function $f(x(t))$ provokes the necessity of a new formula which considers the case when $x(t)$ is a vector.

The objective of this paper is to derive, prove, and present such a new formula for the $n$th total derivative of the composite function $f(\mathbf{x}(t))$ when its argument $\mathbf{x}(t)=\left[x_{1}(t), x_{2}(t), \ldots, x_{r}(t)\right]^{T}$ is a vector with an arbitrary number of components.

2. Generalized formula for the $n$th derivative of a composite function with a vector argument. This section introduces the main result in the form of a theorem and develops the generalized formula on the basis of Faa di Bruno's formula with the help of a specific approach.

THEOREM 2.1. If $f$ and $t$ are scalars, $\mathbf{x}(t)=\left[x_{1}(t), x_{2}(t), \ldots, x_{r}(t)\right]^{T}$ is an $r$-vector and $f(\mathbf{x}(t))$ is a composite function, for which all the necessary derivatives are defined, then

$$
\begin{aligned}
D^{n} f(\mathbf{x}(t))= & \sum_{0} \sum_{1} \sum_{2} \cdots \sum_{n} \frac{n !}{\prod_{i=1}^{n}(i !)^{k_{i}} \prod_{i=1}^{n} \prod_{j=1}^{r} q_{i j} !} \frac{\partial^{k} f}{\partial x_{1}^{p_{1}} \partial x_{2}^{p_{2}} \cdots \partial x_{r}^{p_{r}}} \\
& \times \prod_{i=1}^{n}\left(x_{1}^{(i)}\right)^{q_{i 1}}\left(x_{2}^{(i)}\right)^{q_{i 2}} \cdots\left(x_{r}^{(i)}\right)^{q_{i r}},
\end{aligned}
$$

where the respective sums are over all nonnegative integer solutions of the Diophantine equations, as follows

$$
\begin{aligned}
& \sum_{0} \longrightarrow k_{1}+2 k_{2}+\cdots+n k_{n}=n, \\
& \sum_{1} \longrightarrow q_{11}+q_{12}+\cdots+q_{1 r}=k_{1}, \\
& \sum_{2} \longrightarrow q_{21}+q_{22}+\cdots+q_{2 r}=k_{2}, \\
& \vdots \\
& \sum_{n} \longrightarrow q_{n 1}+q_{n 2}+\cdots+q_{n r}=k_{n},
\end{aligned}
$$


and the differential operator $D=d / d t, p_{j}$-the order of the partial derivative with respect to $x_{j}$, and $k$-the order of the partial derivative are

$$
\begin{aligned}
& p_{j}=q_{1 j}+q_{2 j}+\cdots+q_{n j}, \quad j=1,2, \ldots, r \\
& k=p_{1}+p_{2}+\cdots+p_{r}=k_{1}+k_{2}+\cdots+k_{n} .
\end{aligned}
$$

Proof. If the notation $D_{i}=\partial_{x} x^{(i)}$ is introduced with $\partial_{x}=\partial / \partial x$ and $\left(\partial_{x}\right)^{i}=\partial^{i} / \partial x^{i}$ then, since always $k=k_{1}+k_{2}+\cdots+k_{n}$ the operator for partial differentiation can be represented as $\partial^{k} / \partial x^{k}=\partial^{k} / \partial x^{k_{1}+k_{2}+\cdots+k_{n}}$ and therefore it can be inserted in the parentheses, so formula (1.1) can be written as follows:

$$
f_{t}^{(n)}=\sum \frac{n !\left(\partial_{x} x^{(1)}\right)^{k_{1}}\left(\partial_{x} x^{(2)}\right)^{k_{2}} \cdots\left(\partial_{x} x^{(n)}\right)^{k_{n}} f}{k_{1} ! k_{2} ! \cdots k_{n} !(1 !)^{k_{1}}(2 !)^{k_{2}} \cdots(n !)^{k_{n}}}
$$

or

$$
f_{t}^{(n)}=\sum \frac{n ! D_{1}^{k_{1}} D_{2}^{k_{2}} \cdots D_{n}^{k_{n}} f}{k_{1} ! k_{2} ! \cdots k_{n} !(1 !)^{k_{1}}(2 !)^{k_{2}} \cdots(n !)^{k_{n}}} .
$$

The difference between this record and (1.1) is that the partial derivatives (1.2) and the nonlinear functions (1.4) remain hidden in the operators $D_{i}^{k_{i}}$ and are not shown explicitly. In accordance with formula (1.1), the sequential derivatives $f_{t}^{(i)}, i=1,2,3, \ldots$ are functions with an increasing number of arguments

$$
\begin{aligned}
f_{t}^{(1)} & =f_{t}^{(1)}(x, \dot{x}), \\
f_{t}^{(2)} & =f_{t}^{(2)}(x, \dot{x}, \ddot{x}), \\
f_{t}^{(3)} & =f_{t}^{(3)}(x, \dot{x}, \ddot{x}, \ddot{x}), \\
\vdots & \\
f_{t}^{(n)} & =f_{t}^{(n)}\left(x, \dot{x}, \ddot{x}, \ldots, x^{(n)}\right) .
\end{aligned}
$$

The sequential derivatives $f_{t}^{(i)}, i=1,2,3, \ldots$ can be obtained by considering (2.6) and applying the rule of the first total derivative of a composite function with an arbitrary number of arguments:

$$
\begin{aligned}
f_{t}^{(1)} & =\left(\partial_{x} \dot{x}\right) f=D_{1} f, \\
f_{t}^{(2)} & =\left(\partial_{x} \dot{x}+\partial_{\dot{x}} \ddot{x}\right) f_{t}^{(1)}=\left[\left(\partial_{x} \dot{x}\right)^{2}+\partial_{x} \ddot{x}\right] f=\left(D_{1}^{2}+D_{2}\right) f, \\
f_{t}^{(3)} & =\left(\partial_{x} \dot{x}+\partial_{\dot{x}} \ddot{x}+\partial_{\ddot{x}} \ddot{x}\right) f_{t}^{(2)}=\left[\left(\partial_{x} \dot{x}\right)^{3}+3 \partial_{x} \dot{x} \partial_{x} \ddot{x}+\partial_{x} \ddot{x}\right] f \\
& =\left(D_{1}^{3}+3 D_{1} D_{2}+D_{3}\right) f, \\
f_{t}^{(4)} & =\left(\partial_{x} \dot{x}+\partial_{\dot{x}} \ddot{x}+\partial_{\ddot{x}} \ddot{x}+\partial_{\ddot{x}} x^{(4)}\right) f_{t}^{(3)} \\
& =\left[\left(\partial_{x} \dot{x}\right)^{4}+6\left(\partial_{x} \dot{x}\right)^{2} \partial_{x} \ddot{x}+4 \partial_{x} \dot{x} \partial_{x} \ddot{x}+3\left(\partial_{x} \ddot{x}\right)^{2}+\partial_{x} x^{(4)}\right] f \\
& =\left(D_{1}^{4}+6 D_{1}^{2} D_{2}+4 D_{1} D_{3}+3 D_{2}^{2}+D_{4}\right) f,
\end{aligned}
$$


If the third derivative $f_{t}^{(3)}$ is developed in detail, then the expressions

$$
\left(\partial_{\dot{x}} \ddot{x} \partial_{x} \ddot{x}\right) f, \quad\left(\partial_{\ddot{x}} \ddot{x} \partial_{x} \dot{x}\right) f
$$

will be obtained. They give null results because the function $f(x(t))$ does not depend on $\dot{x}$ and $\ddot{x}$ respectively. Such cases of dropping out of some terms from the final result are observed in the fourth and the next derivatives.

As mentioned in the introduction, it is proved in a number of ways, that the general description of these derivatives is given by the formula of Faa di Bruno (1.1).

Now, let $\mathbf{x}$ be the $r$-vector

$$
\mathbf{x}(t)=\left[x_{1}(t), x_{2}(t), \ldots, x_{r}(t)\right]^{T},
$$

respectively,

$$
\partial_{x}=\frac{\partial}{\partial \mathbf{x}}=\left[\frac{\partial}{\partial x_{1}}, \frac{\partial}{\partial x_{2}}, \ldots, \frac{\partial}{\partial x_{r}}\right]^{T}
$$

be a vector differential operator and $f(\mathbf{x}(t))$ a composite function for which all the necessary derivatives are defined. The derivatives $f_{t}^{(i)}, i=1,2,3, \ldots$ can be obtained via a sequential application of the rule of the first total derivative of a composite function with an arbitrary number of arguments, substitution of the previous derivative and considering the number of its arguments:

$$
\begin{aligned}
f_{t}^{(1)} & =\left(\partial_{x}^{T} \dot{\mathbf{x}}\right) f=\mathbf{D}_{1} f, \\
f_{t}^{(2)} & =\left(\partial_{x}^{T} \dot{\mathbf{x}}+\partial_{\dot{x}}^{T} \ddot{\mathbf{x}}\right) f_{t}^{(1)}=\left[\left(\partial_{x}^{T} \dot{\mathbf{x}}\right)^{2}+\partial_{x}^{T} \ddot{\mathbf{x}}\right] f=\left(\mathbf{D}_{1}^{2}+\mathbf{D}_{2}\right) f, \\
f_{t}^{(3)} & =\left(\partial_{x}^{T} \dot{\mathbf{x}}+\partial_{\dot{x}}^{T} \ddot{\mathbf{x}}+\partial_{\ddot{x}}^{T} \dddot{\mathbf{x}}\right) f_{t}^{(2)}=\left[\left(\partial_{x}^{T} \dot{\mathbf{x}}\right)^{3}+3 \partial_{x}^{T} \dot{\mathbf{x}} \partial_{x}^{T} \ddot{\mathbf{x}}+\partial_{x}^{T} \dddot{\mathbf{x}}\right] f \\
& =\left(\mathbf{D}_{1}^{3}+3 \mathbf{D}_{1} \mathbf{D}_{2}+\mathbf{D}_{3}\right) f, \\
f_{t}^{(4)} & =\left(\partial_{x}^{T} \dot{\mathbf{x}}+\partial_{\dot{x}}^{T} \ddot{\mathbf{x}}+\partial_{\ddot{x}}^{T} \ddot{\mathbf{x}}+\partial_{\ddot{x}}^{T} \mathbf{x}^{(4)}\right) f_{t}^{(3)} \\
& =\left[\left(\partial_{x}^{T} \dot{\mathbf{x}}\right)^{4}+6\left(\partial_{x}^{T} \dot{\mathbf{x}}\right)^{2} \partial_{x}^{T} \ddot{\mathbf{x}}+4 \partial_{x}^{T} \dot{\mathbf{x}} \partial_{x}^{T} \dddot{\mathbf{x}}+3\left(\partial_{x}^{T} \ddot{\mathbf{x}}\right)^{2}+\partial_{x}^{T} \mathbf{x}^{(4)}\right] f \\
& =\left(\mathbf{D}_{1}^{4}+6 \mathbf{D}_{1}^{2} \mathbf{D}_{2}+4 \mathbf{D}_{1} \mathbf{D}_{3}+3 \mathbf{D}_{2}^{2}+\mathbf{D}_{4}\right) f,
\end{aligned}
$$

The process of deriving the derivatives (2.11) is consubstantial with that of the derivatives (2.7), and they have the same operator form, nevertheless in one case the operators are scalar and in the other vector. That is why their general form can be written out in accordance with the formula of Faa di Bruno as

$$
f_{t}^{(n)}=\sum \frac{n !\left(\partial_{x}^{T} \mathbf{x}^{(1)}\right)^{k_{1}}\left(\partial_{x}^{T} \mathbf{x}^{(2)}\right)^{k_{2}} \cdots\left(\partial_{x}^{T} \mathbf{x}^{(n)}\right)^{k_{n}} f}{k_{1} ! k_{2} ! \cdots k_{n} !(1 !)^{k_{1}}(2 !)^{k_{2}} \cdots(n !)^{k_{n}}} .
$$

This step is valid because

- The derivatives (2.11) are obtained via the sequential application of the rule of the first total derivative of a composite function with an arbitrary number of arguments.

- The process of developing the derivatives (2.11) is consubstantial with the process of developing the derivatives (2.7).

The formula (2.12) is a vector form of the $n$th derivative because the operators for 
partial differentiation are in a vector form. Hence, the partial derivatives and the nonlinear functions of the derivatives $\mathbf{x}^{(i)}, i=1,2, \ldots, n$ are not shown explicitly. To obtain the explicit component form of the formula for the $n$th derivative of $f(\mathbf{x}(t))$ it is necessary to raise the polynomials $\left(\partial_{x}^{T} \mathbf{x}^{(i)}\right)^{k_{i}}, i=1,2, \ldots, n$ to the corresponding powers and then to multiply them. For this purpose, it is suitable to number the polynomials in accordance with the derivative which they contain. The polynomial containing $\mathbf{x}^{(1)}$ is the first, the one including $\mathbf{x}^{(2)}$ is the second and so on. Then for the $i$ th polynomial it can be written out

$$
\begin{aligned}
\left(\partial_{x_{1}} x_{1}^{(i)}\right. & \left.+\partial_{x_{2}} x_{2}^{(i)}+\cdots+\partial_{x_{r}} x_{r}^{(i)}\right)^{k_{i}} \\
& =\sum \frac{k_{i} !}{q_{i 1} ! q_{i 2} ! \cdots q_{i r} !}\left(\partial_{x_{1}} x_{1}^{(i)}\right)^{q_{i 1}}\left(\partial_{x_{2}} x_{2}^{(i)}\right)^{q_{i 2}} \cdots\left(\partial_{x_{r}} x_{r}^{(i)}\right)^{q_{i r}} .
\end{aligned}
$$

The sum is over all nonnegative integer solutions of the Diophantine equation $q_{i 1}+$ $q_{i 2}+\cdots+q_{i r}=k_{i}$. Here, the powers $q_{i j}$ are numbered with index $i$-number of the polynomial and $j$-number of the component from the $\mathbf{x}$ vector. If the $n$ polynomials in the form (2.13) are multiplied, then the result is

$$
\begin{aligned}
& f_{t}^{(n)}=\sum_{0} \sum_{1} \sum_{2} \cdots \sum_{n} \frac{\partial^{k} f}{\partial x_{1}^{p_{1}} \partial x_{2}^{p_{2}} \cdots \partial x_{r}^{p_{r}}} \\
& \times \frac{n ! k_{1} ! k_{2} ! \cdots k_{n} !}{k_{1} ! k_{2} ! \cdots k_{n} !(1 !)^{k_{1}}(2 !)^{k_{2}} \cdots(n !)^{k_{n}} q_{11} ! q_{12} ! \cdots q_{1 r} ! q_{21} ! q_{22} ! \cdots q_{2 r} ! \cdots q_{n 1} ! q_{n 2} ! \cdots q_{n r} !} \\
& \times\left(x_{1}^{(1)}\right)^{q_{11}}\left(x_{1}^{(2)}\right)^{q_{21}} \cdots\left(x_{1}^{(n)}\right)^{q_{n 1}}\left(x_{2}^{(1)}\right)^{q_{12}}\left(x_{2}^{(2)}\right)^{q_{22}} \cdots\left(x_{2}^{(n)}\right)^{q_{n 2}} \cdots\left(x_{r}^{(1)}\right)^{q_{1 r}} \\
& \times\left(x_{r}^{(2)}\right)^{q_{2 r}} \cdots\left(x_{r}^{(n)}\right)^{q_{n r}}
\end{aligned}
$$

or in concise record

$$
\begin{aligned}
f_{t}^{(n)}= & \sum_{0} \sum_{1} \sum_{2} \cdots \sum_{n} \frac{n !}{\prod_{i=1}^{n}(i !)^{k_{i}} \prod_{i=1}^{n} \prod_{j=1}^{r} q_{i j} !} \frac{\partial^{k} f}{\partial x_{1}^{p_{1}} \partial x_{2}^{p_{2}} \cdots \partial x_{r}^{p_{r}}} \\
& \times \prod_{i=1}^{n}\left(x_{1}^{(i)}\right)^{q_{i 1}}\left(x_{2}^{(i)}\right)^{q_{i 2}} \cdots\left(x_{r}^{(i)}\right)^{q_{i r}},
\end{aligned}
$$

whereas the sums are over all nonnegative integer solutions of the Diophantine equations

$$
\begin{aligned}
& \sum_{0} \longrightarrow k_{1}+2 k_{2}+\cdots+n k_{n}=n, \\
& \sum_{1} \longrightarrow q_{11}+q_{12}+\cdots+q_{1 r}=k_{1}, \\
& \sum_{2} \longrightarrow q_{21}+q_{22}+\cdots+q_{2 r}=k_{2}, \\
& \vdots \\
& \sum_{n} \longrightarrow q_{n 1}+q_{n 2}+\cdots+q_{n r}=k_{n},
\end{aligned}
$$


with $p_{j}$ being the order of the partial derivative with respect to $x_{j}$ and $k$-the order of the partial derivative

$$
\begin{aligned}
& p_{j}=q_{1 j}+q_{2 j}+\cdots+q_{n j}, \quad j=1,2, \ldots, r, \\
& k=p_{1}+p_{2}+\cdots+p_{r}=k_{1}+k_{2}+\cdots+k_{n} .
\end{aligned}
$$

This result coincides with formula (2.1), which completes the proof.

Consistency. Formula (2.1) is an explicit component form of the $n$th derivative of the composite function $f(\mathbf{x}(t))$. It is a straightforward generalization of the formula of Faa di Bruno when the argument $\mathbf{x}(t)$ is a vector. In this sense, Faa di Bruno's formula is a special case of formula (2.1) when $r=1$, and therefore $\mathbf{x}(t)=x_{1}(t)$. Then, the Diophantine equations (2.2) and the variables (2.3) take the form

$$
\begin{aligned}
& \sum_{0} \longrightarrow k_{1}+2 k_{2}+\cdots+n k_{n}=n \\
& \sum_{1} \longrightarrow q_{11}=k_{1} \\
& \sum_{2} \longrightarrow q_{21}=k_{2} \\
& \vdots \\
& \sum_{n} \longrightarrow q_{n 1}=k_{n}, \\
& p_{1}=q_{11}+q_{21}+\cdots+q_{n 1}, \\
& k=p_{1}=k_{1}+k_{2}+\cdots+k_{n} .
\end{aligned}
$$

In this case, the expression (2.1) reads

$$
\begin{aligned}
D^{n} f & \left(x_{1}(t)\right) \\
& =\sum_{0} \frac{n !}{k_{1} ! k_{2} ! \cdots k_{n} !(1 !)^{k_{1}}(2 !)^{k_{2}} \cdots(n !)^{k_{n}}} \frac{\partial^{k} f}{\partial x_{1}^{k}}\left(x_{1}^{(1)}\right)^{k_{1}}\left(x_{1}^{(2)}\right)^{k_{2}} \cdots\left(x_{1}^{(n)}\right)^{k_{n}},
\end{aligned}
$$

which is actually the formula of Faa di Bruno when $x=x_{1}$.

The use of formula (2.1) requires the solution of the system of linear Diophantine equations (2.2). When the order of the derivative considered is low and the number of arguments $r$ is small, then these equations can be easily solved manually. It is well known that they do not have general analytic solution. So, when we deal with higher order derivatives and a bigger number of arguments, an efficient tool for solving such equations is needed. Clausen and Fortenbacher [2] have proposed algorithms and an efficient PASCAL program for solving a wide class of linear Diophantine equations, including equations of the type (2.2). The solution of the system of linear Diophantine equations can be obtained smoothly with the help of this program.

3. Example. The fourth derivative of the function

$$
f\left(x_{1}(t), x_{2}(t)\right)=e^{x_{1}(t) x_{2}(t)}
$$


will be considered as an illustration example for formula (2.1). First, the fourth derivative of $f\left(x_{1}(t), x_{2}(t)\right)$ will be considered in order to show its general structure, and then it will be applied over the function $e^{x_{1}(t) x_{2}(t)}$. In this case, formula (2.1) has the form

$$
\begin{aligned}
& f\left(x_{1}(t), x_{2}(t)\right)^{(4)} \\
& \quad=\sum_{0} \sum_{1} \sum_{2} \sum_{3} \sum_{4} \frac{4 !}{\prod_{i=1}^{4}(i !)^{k_{i}} \prod_{i=1}^{4} \prod_{j=1}^{2} q_{i j} !} \frac{\partial^{k} f}{\partial x_{1}^{p_{1}} \partial x_{2}^{p_{2}}} \prod_{i=1}^{4}\left(x_{1}^{(i)}\right)^{q_{i 1}}\left(x_{2}^{(i)}\right)^{q_{i 2}}
\end{aligned}
$$

and the equations (2.2),

$$
\begin{aligned}
& \sum_{0} \rightarrow k_{1}+2 k_{2}+3 k_{3}+4 k_{4}=4 \\
& \sum_{1} \rightarrow q_{11}+q_{12}=k_{1} \\
& \sum_{2} \rightarrow q_{21}+q_{22}=k_{2} \\
& \sum_{3} \longrightarrow q_{31}+q_{32}=k_{3} \\
& \sum_{4} \rightarrow q_{41}+q_{42}=k_{4}
\end{aligned}
$$

TABLE 3.1.

\begin{tabular}{ccccc}
\hline No. & $k_{1}$ & $k_{2}$ & $k_{3}$ & $k_{4}$ \\
\hline 1 & 4 & 0 & 0 & 0 \\
\hline 2 & 2 & 1 & 0 & 0 \\
\hline 3 & 1 & 0 & 1 & 0 \\
\hline 4 & 0 & 2 & 0 & 0 \\
\hline 5 & 0 & 0 & 0 & 1 \\
\hline
\end{tabular}

The solutions of equation (3.3) are shown in Table 3.1, and they form the most external loop of the sums. These five solutions define the respective five sets of combinations of solutions of the equations (3.4) as shown in Table 3.2. The five solutions of the main Diophantine equation (3.3), corresponding to $\sum_{0}$, are listed in the first column of this table. Each of those solutions defines the Diophantine equations (3.4), corresponding to $\sum_{i}, i=1,2,3,4$, which are shown in the second column. All possible combinations of solutions of the latter equations are shown in the remaining columns. The sums $p_{1}=q_{11}+q_{21}+q_{31}+q_{41}$ and $p_{2}=q_{12}+q_{22}+q_{32}+q_{42}$ determine the order of the partial derivatives with respect to $x_{1}$ and $x_{2}$. So, the fourth total derivative of the function $f\left(x_{1}(t), x_{2}(t)\right)$ can be written out in general form on the basis of formula (3.2) and Table 3.2, 


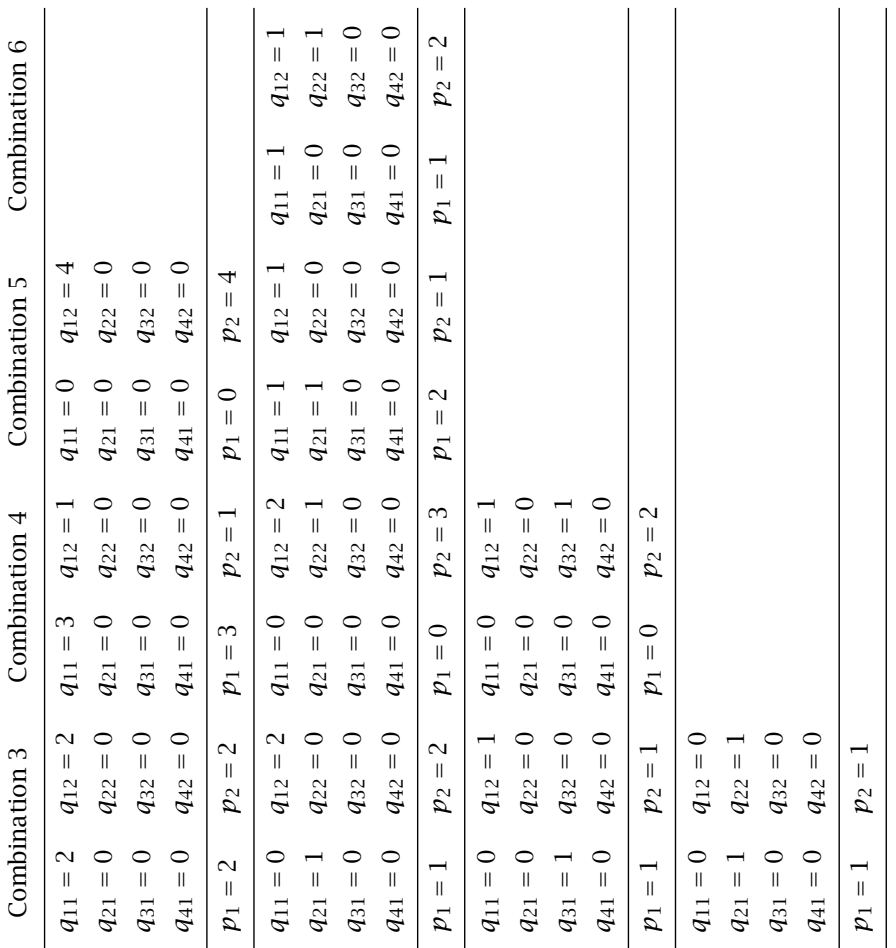

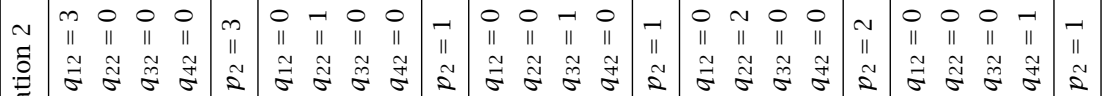

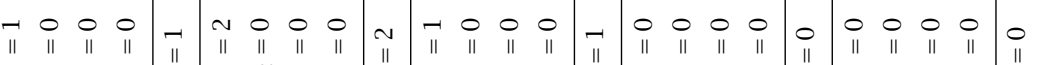

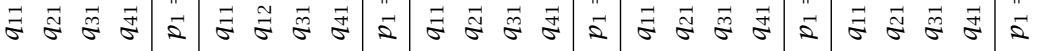

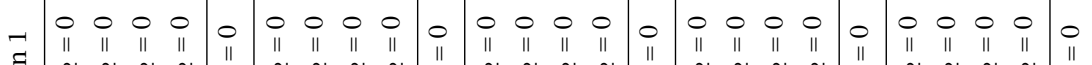

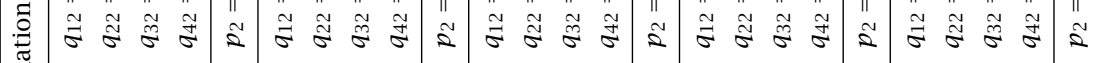

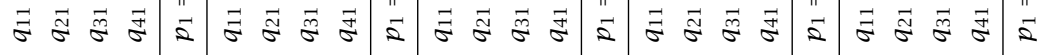

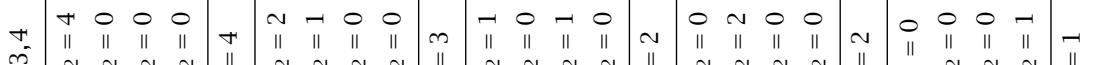

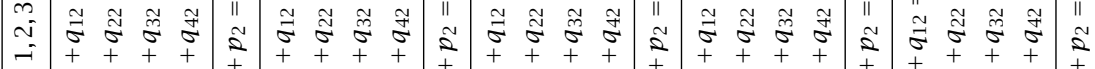

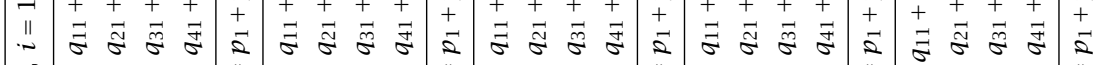
ผี่

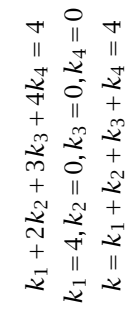

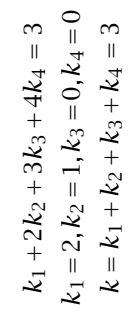

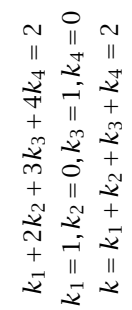

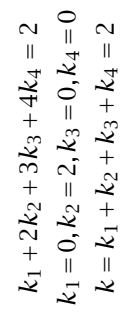

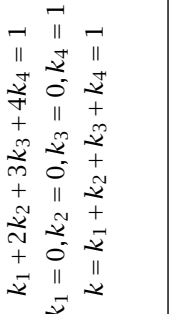




$$
\begin{aligned}
& f\left(x_{1}(t), x_{2}(t)\right)^{(4)}=\frac{4 !}{(1 !)^{4}(2 !)^{0}(3 !)^{0}(4 !)^{0} 4 ! 0 ! 0 ! 0 ! 0 ! 0 ! 0 ! 0 !} \frac{\partial^{4} f}{\partial x_{1}^{4}} \dot{x}_{1}^{4} \\
& +\frac{4 !}{(1 !)^{4}(2 !)^{0}(3 !)^{0}(4 !)^{0} 1 ! 0 ! 0 ! 0 ! 3 ! 0 ! 0 ! 0 !} \frac{\partial^{4} f}{\partial x_{1} \partial x_{2}^{3}} \dot{x}_{1} \dot{x}_{2}^{3} \\
& +\frac{4 !}{\left.(1 !)^{4}(2 !)^{0}(3 !)^{0}(4 !)\right)^{0} 2 ! 0 ! 0 ! 0 ! 2 ! 0 ! 0 ! 0 !} \frac{\partial^{4} f}{\partial x_{1}^{2} \partial x_{2}^{2}} \dot{x}_{1}^{2} \dot{x}_{2}^{2} \\
& +\frac{4 !}{(1 !)^{4}(2 !)^{0}(3 !)^{0}(4 !)^{0} 3 ! 0 ! 0 ! 0 ! 1 ! 0 ! 0 ! 0 !} \frac{\partial^{4} f}{\partial x_{1}^{3} \partial x_{2}} \dot{x}_{1}^{3} \dot{x}_{2} \\
& +\frac{4 !}{(1 !)^{4}(2 !)^{0}(3 !)^{0}(4 !)^{0} 0 ! 0 ! 0 ! 0 ! 4 ! 0 ! 0 ! 0 !} \frac{\partial^{4} f}{\partial x_{2}^{4}} \dot{x}_{2}^{4} \\
& +\frac{4 !}{(1 !)^{2}(2 !)^{1}(3 !)^{0}(4 !)^{0} 2 ! 1 ! 0 ! 0 ! 0 ! 0 ! 0 ! 0 !} \frac{\partial^{3} f}{\partial x_{1}^{3}} \dot{x}_{1}^{2} \ddot{x}_{1} \\
& +\frac{4 !}{(1 !)^{2}(2 !)^{1}(3 !)^{0}(4 !)^{0} 2 ! 0 ! 0 ! 0 ! 0 ! 1 ! 0 ! 0 !} \frac{\partial^{3} f}{\partial x_{1}^{2} \partial x_{2}} \dot{x}_{1}^{2} \ddot{x}_{2} \\
& +\frac{4 !}{(1 !)^{2}(2 !)^{1}(3 !)^{0}(4 !)^{0} 0 ! 1 ! 0 ! 0 ! 2 ! 0 ! 0 ! 0 !} \frac{\partial^{3} f}{\partial x_{1} \partial x_{2}^{2}} \ddot{x}_{1} \dot{x}_{2}^{2} \\
& +\frac{4 !}{(1 !)^{2}(2 !)^{1}(3 !)^{0}(4 !)^{0} 0 ! 0 ! 0 ! 0 ! 2 ! 1 ! 0 ! 0 !} \frac{\partial^{3} f}{\partial x_{2}^{3}} \dot{x}_{2}^{2} \ddot{x}_{2} \\
& +\frac{4 !}{(1 !)^{2}(2 !)^{1}(3 !)^{0}(4 !)^{0} 1 ! 1 ! 0 ! 0 ! 1 ! 0 ! 0 ! 0 !} \frac{\partial^{3} f}{\partial x_{1}^{2} \partial x_{2}} \dot{x}_{1} \ddot{x}_{1} \dot{x}_{2} \\
& +\frac{4 !}{(1 !)^{2}(2 !)^{1}(3 !)^{0}(4 !)^{0} 1 ! 0 ! 0 ! 0 ! 1 ! 1 ! 0 ! 0 !} \frac{\partial^{3} f}{\partial x_{1} \partial x_{2}^{2}} \dot{x}_{1} \dot{x}_{2} \ddot{x}_{2} \\
& +\frac{4 !}{(1 !)^{1}(2 !)^{0}(3 !)^{1}(4 !)^{0} 1 ! 0 ! 1 ! 0 ! 0 ! 0 ! 0 ! 0 !} \frac{\partial^{2} f}{\partial x_{1}^{2}} \dot{x}_{1} \dddot{x}_{1} \\
& +\frac{4 !}{(1 !)^{1}(2 !)^{0}(3 !)^{1}(4 !)^{0} 1 ! 0 ! 0 ! 0 ! 0 ! 0 ! 1 ! 0 !} \frac{\partial^{2} f}{\partial x_{1} \partial x_{2}} \dot{x}_{1} \dddot{x}_{2} \\
& +\frac{4 !}{(1 !)^{1}(2 !)^{0}(3 !)^{1}(4 !)^{0} 0 ! 0 ! 1 ! 0 ! 1 ! 0 ! 0 ! 0 !} \frac{\partial^{2} f}{\partial x_{1} \partial x_{2}} \dddot{x}_{1} \dot{x}_{2} \\
& +\frac{4 !}{(1 !)^{1}(2 !)^{0}(3 !)^{1}(4 !)^{0} 0 ! 0 ! 0 ! 0 ! 1 ! 0 ! 1 ! 0 !} \frac{\partial^{2} f}{\partial x_{2}^{2}} \dot{x}_{2} \dddot{x}_{2} \\
& +\frac{4 !}{(1 !)^{0}(2 !)^{2}(3 !)^{0}(4 !)^{0} 0 ! 2 ! 0 ! 0 ! 0 ! 0 ! 0 ! 0 !} \frac{\partial^{2} f}{\partial x_{1}^{2}} \ddot{x}_{1}^{2} \\
& +\frac{4 !}{(1 !)^{0}(2 !)^{2}(3 !)^{0}(4 !)^{0} 0 ! 0 ! 0 ! 0 ! 0 ! 2 ! 0 ! 0 !} \frac{\partial^{2} f}{\partial x_{2}^{2}} \ddot{x}_{2}^{2} \\
& +\frac{4 !}{(1 !)^{0}(2 !)^{2}(3 !)^{0}(4 !)^{0} 0 ! 1 ! 0 ! 0 ! 0 ! 1 ! 0 ! 0 !} \frac{\partial^{2} f}{\partial x_{1} \partial x_{2}} \ddot{x}_{1} \ddot{x}_{2} \\
& +\frac{4 !}{(1 !)^{0}(2 !)^{0}(3 !)^{0}(4 !)^{1} 0 ! 0 ! 0 ! 1 ! 0 ! 0 ! 0 ! 0 !} \frac{\partial f}{\partial x_{1}} x_{1}^{(4)} \\
& +\frac{4 !}{(1 !)^{0}(2 !)^{0}(3 !)^{0}(4 !)^{1} 0 ! 0 ! 0 ! 0 ! 0 ! 0 ! 0 ! 1 !} \frac{\partial f}{\partial x_{2}} x_{2}^{(4)}
\end{aligned}
$$


or finally

$$
\begin{aligned}
f\left(x_{1}(t), x_{2}(t)\right)^{(4)}= & \frac{\partial^{4} f}{\partial x_{1}^{4}} \dot{x}_{1}^{4}+4 \frac{\partial^{4} f}{\partial x_{1} \partial x_{2}^{3}} \dot{x}_{1} \dot{x}_{2}^{3}+6 \frac{\partial^{4} f}{\partial x_{1}^{2} \partial x_{2}^{2}} \dot{x}_{1}^{2} \dot{x}_{2}^{2}+4 \frac{\partial^{4} f}{\partial x_{1}^{3} \partial x_{2}} \dot{x}_{1}^{3} \dot{x}_{2} \\
& +\frac{\partial^{4} f}{\partial x_{2}^{4}} \dot{x}_{2}^{4}+6 \frac{\partial^{3} f}{\partial x_{1}^{3}} \dot{x}_{1}^{2} \ddot{x}_{1}+6 \frac{\partial^{3} f}{\partial x_{1}^{2} \partial x_{2}} \dot{x}_{1}^{2} \ddot{x}_{2}+6 \frac{\partial^{3} f}{\partial x_{1} \partial x_{2}^{2}} \ddot{x}_{1} \dot{x}_{2}^{2} \\
& +6 \frac{\partial^{3} f}{\partial x_{2}^{3}} \dot{x}_{2}^{2} \ddot{x}_{2}+12 \frac{\partial^{3} f}{\partial x_{1}^{2} \partial x_{2}} \dot{x}_{1} \ddot{x}_{1} \dot{x}_{2}+12 \frac{\partial^{3} f}{\partial x_{1} \partial x_{2}^{2}} \dot{x}_{1} \dot{x}_{2} \ddot{x}_{2} \\
& +4 \frac{\partial^{2} f}{\partial x_{1}^{2}} \dot{x}_{1} \dddot{x}_{1}+4 \frac{\partial^{2} f}{\partial x_{1} \partial x_{2}} \dot{x}_{1} \dddot{x}_{2}+4 \frac{\partial^{2} f}{\partial x_{1} \partial x_{2}} \dddot{x}_{1} \dot{x}_{2}+4 \frac{\partial^{2} f}{\partial x_{2}^{2}} \dot{x}_{2} \dddot{x}_{2} \\
& +3 \frac{\partial^{2} f}{\partial x_{1}^{2}} \ddot{x}_{1}^{2}+3 \frac{\partial^{2} f}{\partial x_{2}^{2}} \ddot{x}_{2}^{2}+6 \frac{\partial^{2} f}{\partial x_{1} \partial x_{2}} \ddot{x}_{1} \ddot{x}_{2}+\frac{\partial f}{\partial x_{1}} x_{1}^{(4)}+\frac{\partial f}{\partial x_{2}} x_{2}^{(4)} .
\end{aligned}
$$

Specifically, for the function $e^{x_{1}(t) x_{2}(t)}$ the result is

$$
\begin{aligned}
\left(e^{x_{1} x_{2}}\right)^{(4)}= & e^{x_{1} x_{2}}\left[x_{2}^{4} \dot{x}_{1}^{4}+4\left(x_{1}^{3} x_{2}+3 x_{1}^{2}\right) \dot{x}_{1} \dot{x}_{2}^{3}+6\left(x_{1}^{2} x_{2}^{2}+4 x_{1} x_{2}+2\right) \dot{x}_{1}^{2} \dot{x}_{2}^{2}\right. \\
& +4\left(x_{1} x_{2}^{3}+3 x_{2}^{2}\right) \dot{x}_{1}^{3} \dot{x}_{2}+x_{1}^{4} \dot{x}_{2}^{4}+6 x_{2}^{3} \dot{x}_{1}^{2} \ddot{x}_{1}+6\left(x_{1} x_{2}^{2}+2 x_{2}\right) \dot{x}_{1}^{2} \ddot{x}_{2} \\
& +6\left(x_{1}^{2} x_{2}+2 x_{1}\right) \ddot{x}_{1} \dot{x}_{2}^{2}+6 x_{1}^{3} \dot{x}_{2}^{2} \ddot{x}_{2}+12\left(x_{1} x_{2}^{2}+2 x_{2}\right) \dot{x}_{1} \ddot{x}_{1} \dot{x}_{2} \\
& +12\left(x_{1}^{2} x_{2}+2 x_{1}\right) \dot{x}_{1} \dot{x}_{2} \ddot{x}_{2}+4 x_{2}^{2} \dot{x}_{1} \dddot{x}_{1}+4\left(x_{1} x_{2}+1\right) \dot{x}_{1} \dddot{x}_{2} \\
& +4\left(x_{1} x_{2}+1\right) \dddot{x}_{1} \dot{x}_{2}+4 x_{1}^{2} \dot{x}_{2} \dddot{x}_{2}+3 x_{2}^{2} \ddot{x}_{1}^{2}+3 x_{1}^{2} \ddot{x}_{2}^{2} \\
& \left.+6\left(x_{1} x_{2}+1\right) \ddot{x}_{1} \ddot{x}_{2}+x_{2} x_{1}^{(4)}+x_{1} x_{2}^{(4)}\right] .
\end{aligned}
$$

A comparison has been made with the result obtained from the REDUCE program for symbolic computations with the purpose of checking it up. It has given the same expression for the fourth total derivative of the function $e^{x_{1}(t) x_{2}(t)}$.

4. Conclusions. The formula proposed in this paper is an explicit component form of the $n$th total derivative of a composite function of a vector argument with an arbitrary number of components. It is a straightforward generalization of the formula of Faa di Bruno, and it is consistent with the latter, indeed. In this sense, the formula of Faa di Bruno is a special case of formula (2.1) and can be obtained from it when $r=1$ and therefore $\mathbf{x}(t)=x_{1}(t)$. The number of nested sums in the new formula is $n+1$ against 1 in formula (1.1) which corresponds to the number of Diophantine equations. The greater complexity is the cost of the ability to manage composite functions of a multivariable argument.

\section{REFERENCES}

[1] E. T. Bell, Partition polynomials, Ann. Math. 29 (1927), 38-46.

[2] M. Clausen and A. Fortenbacher, Efficient solution of linear Diophantine equations, J. Symbolic Comput. 8 (1989), no. 1-2, 201-216. MR 90i:11151. Zbl 674.10011.

[3] L. Comtet, Advanced Combinatorics, D. Reidel Publishing Co., Dordrecht, 1974. MR 57\#124. Zbl 283.05001.

[4] C. Jordan, Calculus of Finite Differences, Chelsea Publishing Co., New York, 1965. MR 32\#1463. Zbl 154.33901. 
[5] J. Riordan, An Introduction to Combinatorial Analysis, John Wiley \& Sons Inc., New York, 1958. MR 20\#3077. Zbl 078.00805.

[6] S. Roman, The formula of Faa di Bruno, Amer. Math. Monthly 87 (1980), no. 10, 805-809. MR 82d:26003. Zbl 513.05009.

Rumen L. Mishkov: Str. Asen Zlatarov 32A, Plovdiv 4000, Bulgaria

E-mail address: roumen@netvisio.net 


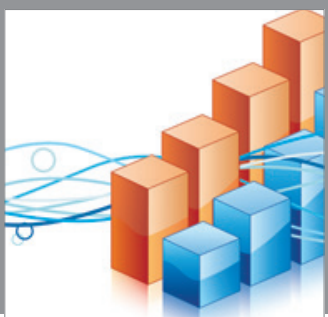

Advances in

Operations Research

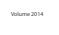

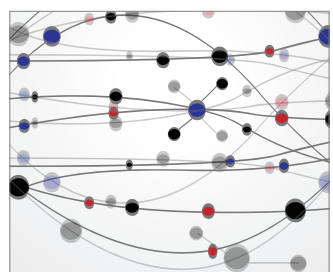

\section{The Scientific} World Journal
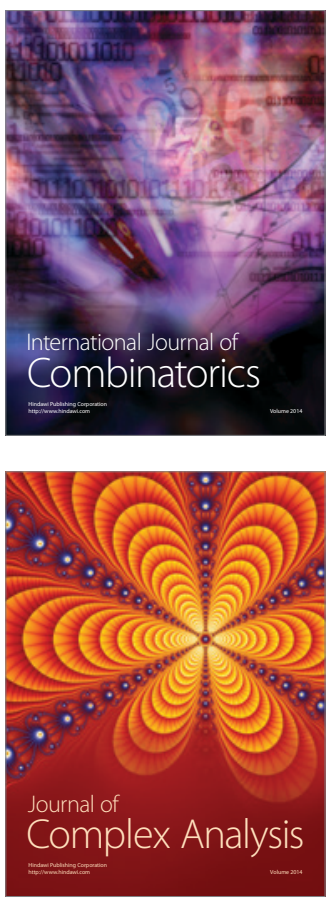

International Journal of

Mathematics and

Mathematical

Sciences
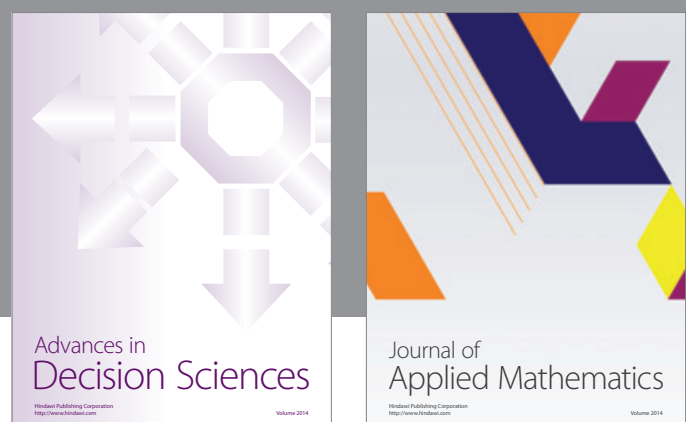

Journal of

Applied Mathematics
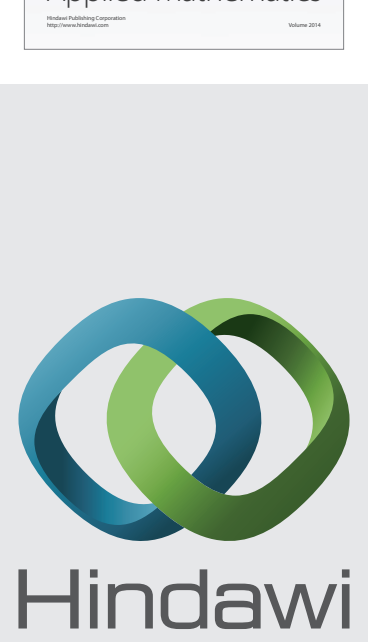

Submit your manuscripts at http://www.hindawi.com
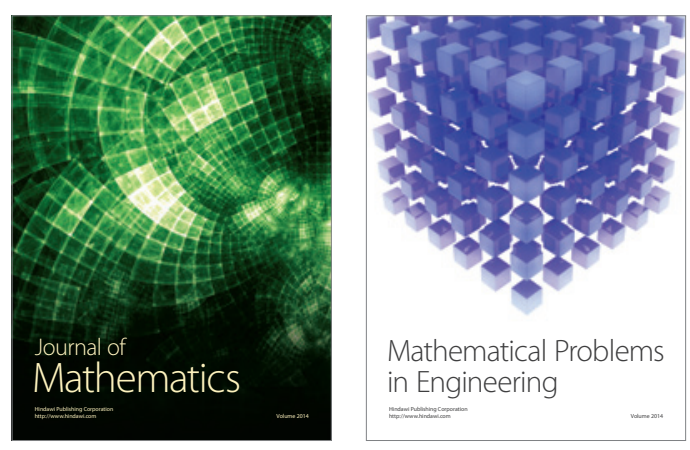

Mathematical Problems in Engineering
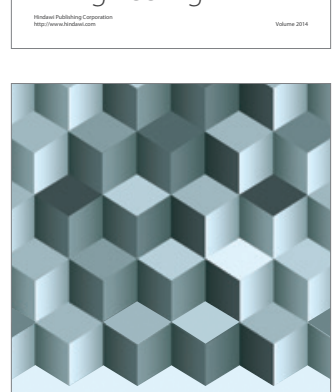

Journal of

Function Spaces
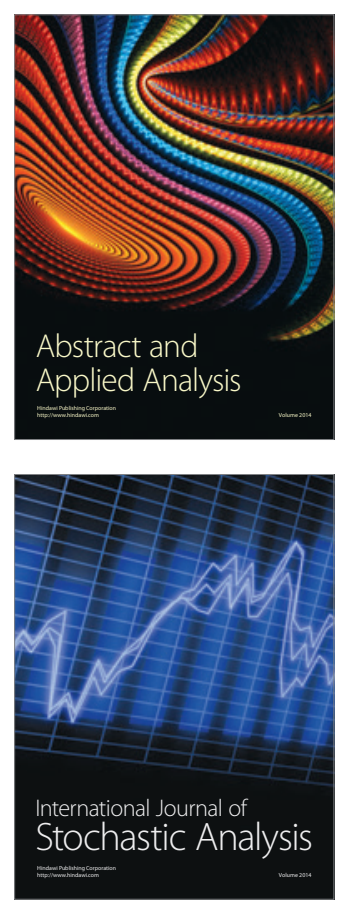

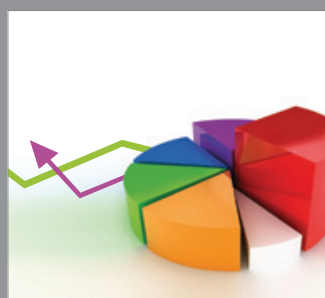

ournal of

Probability and Statistics

Promensencen
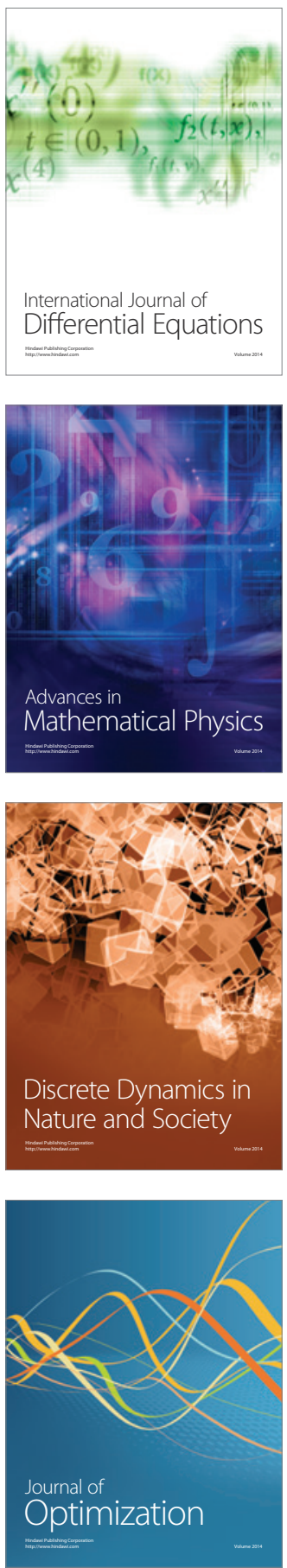